\title{
Free groups acting without fixed points on rational spheres
}

by

\author{
KenZi Satô (Yokohama)
}

For every positive rational number $q$, we find a free group of rotations of rank 2 acting on $\left(\sqrt{q} \mathbb{S}^{2}\right) \cap \mathbb{Q}^{3}$ whose all elements distinct from the identity have no fixed point.

Introduction. The following conjecture raised by Professor J. Mycielski was proved in [Sa1]:

The subgroup $\left\langle\mu_{1}, \nu_{1}\right\rangle$ of the rational special orthogonal group $S O_{3}(\mathbb{Q})=$ $\left\{\phi \in \operatorname{Mat}(3,3 ; \mathbb{Q}):{ }^{t} \phi \cdot \phi=\mathrm{id}, \operatorname{det} \phi=1\right\}$ is a free group of rank 2 whose non-trivial elements have no fixed point on the rational unit sphere $\mathbb{S}^{2} \cap \mathbb{Q}^{3}$ $=\left\{\vec{v} \in \mathbb{Q}^{3}:|\vec{v}|=1\right\}$, where $\left\langle\mu_{1}, \nu_{1}\right\rangle$ is the group generated by

$$
\mu_{1}=\frac{1}{7}\left(\begin{array}{ccc}
6 & 2 & 3 \\
2 & 3 & -6 \\
-3 & 6 & 2
\end{array}\right) \quad \text { and } \quad \nu_{1}=\frac{1}{7}\left(\begin{array}{ccc}
2 & -6 & 3 \\
6 & 3 & 2 \\
-3 & 2 & 6
\end{array}\right) \text {. }
$$

In this paper, we consider the same problem about the rational sphere $\left(\sqrt{q} \mathbb{S}^{2}\right) \cap \mathbb{Q}^{3}=\left\{\vec{v} \in \mathbb{Q}^{3}:|\vec{v}|=\sqrt{q}\right\}$ for positive $q \in \mathbb{Q}$. Notice that the rational unit sphere $\mathbb{S}^{2} \cap \mathbb{Q}^{3}$ and the rational sphere $\left(\sqrt{2} \mathbb{S}^{2}\right) \cap \mathbb{Q}^{3}$ are not similar. In particular, $\mathbb{S}^{2} \cap \mathbb{Q}^{3}$ has a trio of pairwise orthogonal vectors

$$
\vec{e}_{0}=\left(\begin{array}{l}
1 \\
0 \\
0
\end{array}\right), \quad \vec{e}_{1}=\left(\begin{array}{l}
0 \\
1 \\
0
\end{array}\right), \quad \vec{e}_{2}=\left(\begin{array}{l}
0 \\
0 \\
1
\end{array}\right),
$$

but $\left(\sqrt{2} \mathbb{S}^{2}\right) \cap \mathbb{Q}^{3}$ does not have such a trio, because, for two orthogonal vectors $\vec{v}, \vec{v}^{\prime} \in\left(\sqrt{2} \mathbb{S}^{2}\right) \cap \mathbb{Q}^{3}$, the vector $\frac{1}{\sqrt{2}} \vec{v} \times \vec{v}^{\prime}$ does not belong to $\mathbb{Q}^{3}$. The purpose of this paper is to prove:

1991 Mathematics Subject Classification: Primary 20E05, 20H05, 20H20; Secondary 15A18, 51F20, 51F25. 
For each positive rational $q, S O_{3}(\mathbb{Q})$ has a free subgroup $\left\langle\mu_{q}, \nu_{q}\right\rangle$ which acts on $\left(\sqrt{q} \mathbb{S}^{2}\right) \cap \mathbb{Q}^{3}$, the rational sphere with radius $\sqrt{q}$, and whose nontrivial elements have no fixed point on $\left(\sqrt{q} \mathbb{S}^{2}\right) \cap \mathbb{Q}^{3}$.

This implies a paradox: $\left(\sqrt{q} \mathbb{S}^{2}\right) \cap \mathbb{Q}^{3}$ has a Hausdorff decomposition, i.e., $\left(\sqrt{q} \mathbb{S}^{2}\right) \cap \mathbb{Q}^{3}$ can be partitioned into three subsets $A, B$, and $C$ such that $A, B, C, A \cup B, B \cup C$, and $C \cup A$ are all congruent by rotations of $\left\langle\mu_{q}, \nu_{q}\right\rangle$ (see e.g. [Sa0; W, Cor. 4.12]). We only have to prove the assertion for each positive integer $q$ which is square-free, in other words, $q$ does not have a prime whose square divides $q$, because the rational spheres $\left(\sqrt{q} \mathbb{S}^{2}\right) \cap \mathbb{Q}^{3}$ and $\left(\sqrt{q^{\prime}} \mathbb{S}^{2}\right) \cap \mathbb{Q}^{3}$ are similar with similitude ratio $\sqrt{q}: \sqrt{q^{\prime}}$ if $\sqrt{q} / \sqrt{q^{\prime}} \in \mathbb{Q}$. Moreover, we can assume $q \neq 1$ from [Sa1].

REMARK. It is possible that rational spheres are empty (e.g., with radius $\sqrt{7}$ ). For given $q$, it is easy to check whether the rational sphere with radius $\sqrt{q}$ is empty or not (see [M, Ch. 20]):

$$
\begin{array}{ll}
\left(\sqrt{q} \mathbb{S}^{2}\right) \cap \mathbb{Q}^{3} \neq \emptyset & \text { if } q \equiv 1,2,3,5, \text { or } 6(\bmod 8), \\
\left(\sqrt{q} \mathbb{S}^{2}\right) \cap \mathbb{Q}^{3}=\emptyset & \text { if } q \equiv 7(\bmod 8) .
\end{array}
$$

For higher dimensional spheres, Professor J. Mycielski raised the following problems (see [Sa1]):

Problem A. For $n \in \mathbb{N}, n$ even, $n \geq 4$, does $S O_{n}(\mathbb{Q})$ have a free nonabelian subgroup $F_{2}$ such that no elements of $F_{2}$ different from the identity have eigenvectors in $\mathbb{Q}^{n}$ ?

Problem B. For $n \in \mathbb{N}, n$ odd, $n \geq 5$, does $S O_{n}(\mathbb{Q})$ have a free nonabelian subgroup $F_{2}$ which acts without non-trivial fixed points on $\mathbb{S}^{n-1} \cap \mathbb{Q}^{n}$ and is such that if $f, g \in F_{2}$ have a common eigenvector in $\mathbb{Q}^{n}$ then $f g=g f$ ?

[Sa2], which gives a free group $\langle\sigma, \tau\rangle$ of $S_{4}(\mathbb{Q})$ acting on $\mathbb{S}^{3}$ without non-trivial fixed points, and [Sa1] answer in the affirmative Problem A for $n \equiv 0(\bmod 4)$ and Problem B for $n \equiv-1(\bmod 4)$. This paper and [Sa2] also answer in the affirmative the following problem for $n \equiv-1(\bmod 4)$ :

Problem $\mathrm{B}^{\prime}$. For a positive rational $q$ and an odd integer $n \geq 5$, does $S O_{n}(\mathbb{Q})$ have a free non-abelian subgroup $F_{2}$ which acts without non-trivial fixed points on $\left(\sqrt{q} \mathbb{S}^{n-1}\right) \cap \mathbb{Q}^{n}$ and is such that if $f, g \in F_{2}$ have a common eigenvector in $\mathbb{Q}^{n}$ then $f g=g f$ ?

For $n=3$, similar problems for more general surfaces, which the referee of this paper suggested, can be considered: 
Problem C. Is there a free subgroup of rank 2 of the group $\{\phi \in$ $\operatorname{Mat}(3,3 ; \mathbb{Q}):{ }^{t} \phi \cdot \Lambda \cdot \phi=\Lambda$, $\left.\operatorname{det} \phi=1\right\}$ acting on the rational surface

$$
\left\{\left(\begin{array}{l}
x \\
y \\
z
\end{array}\right) \in \mathbb{Q}^{3}: \alpha x^{2}+\beta y^{2}+\gamma z^{2}=q\right\}
$$

without non-trivial fixed points (where $\left.\Lambda=\left(\begin{array}{ccc}\alpha & 0 & 0 \\ 0 & \beta & 0 \\ 0 & 0 & \gamma\end{array}\right)\right)$ ?

Preliminaries. Let $q$ be a positive and square-free integer distinct from 1. The following two lemmas enable us to find integers $p$ and $b$ such that $p$ is an odd prime divisor of $1+b^{2}$ but not of $q$, and $q$ is a quadratic non-residue to the modulus $p$.

Lemma 0. For such an integer $q$, there exists an odd prime $p$ such that $q$ is not divisible by $p$ and

$$
\left(\frac{-1}{p}\right)=1 \quad \text { and } \quad\left(\frac{q}{p}\right)=-1,
$$

where (:) is Legendre's symbol.

Proof. This is a special case of [H, Satz 147]: "Let $a_{1}, a_{2}, \ldots, a_{r}$ be integers such that: a product of powers

$$
a_{1}^{u_{1}} a_{2}^{u_{2}} \ldots a_{r}^{u_{r}}
$$

is the square of an integer (if and) only if all $u_{i}$ 's are even. Furthermore, let $c_{1}, c_{2}, \ldots, c_{r}$ be arbitrary one of \pm 1 . Then there exist infinitely many primes $p$ which satisfy the condition

$$
\left(\frac{a_{i}}{p}\right)=c_{i} \quad \text { for } i=1,2, \ldots, r . "
$$

LEMMA 1. For such a prime $p$, there exists an integer $b$ such that $1+b^{2}$ $\equiv 0(\bmod p)$.

Proof. This is obvious from $\left(\frac{-1}{p}\right)=1$.

Using such an integer $b$, let

$$
\mu_{q}=\frac{1}{1+b^{2}}\left(\begin{array}{ccc}
1+b^{2} & 0 & 0 \\
0 & 1-b^{2} & -2 b \\
0 & 2 b & 1-b^{2}
\end{array}\right)
$$

and

$$
\nu_{q}=\frac{1}{1+b^{2}}\left(\begin{array}{ccc}
1-b^{2} & -2 b & 0 \\
2 b & 1-b^{2} & 0 \\
0 & 0 & 1+b^{2}
\end{array}\right)
$$


There are group isomorphisms

$$
S(\mathbb{H}) /\{ \pm 1\} \underset{\cong}{\stackrel{\sigma}{\longrightarrow}} S O\left(\mathbb{H}_{0}\right) \stackrel{\mathfrak{m}}{\cong} S O_{3}(\mathbb{R}),
$$

where $S(\mathbb{H})$ is the group of quaternions $h$ whose norm $|h|$ is equal to 1 in the Hamilton quaternion field $\mathbb{H}$ and $S O\left(\mathbb{H}_{0}\right)$ is the group of all linear isometries with determinant 1 of all pure quaternions $\mathbb{H}_{0}$ onto itself. The left isomorphism $\sigma$ sends $\pm h$ to the isometry $\mathbb{H}_{0} \ni h^{\prime} \mapsto h h^{\prime} h^{-1} \in \mathbb{H}_{0}$. On the other hand, the linear map $\iota: \mathbb{H}_{0} \rightarrow \mathbb{R}^{3}$, which takes the basis $I, J$, and $K$ of $\mathbb{H}_{0}$ (with $I J=K, J K=I, K I=J$, and $I^{2}=J^{2}=K^{2}=-1$ ) to the standard basis $\vec{e}_{0}, \vec{e}_{1}$, and $\vec{e}_{2}$ of $\mathbb{R}^{3}$ respectively, gives the right isomorphism $\mathfrak{m}: \kappa \mapsto \iota \circ \kappa \circ \iota^{-1}$. So we have $S(\mathbb{H}) /\{ \pm 1\} \stackrel{\mathfrak{m} \circ \sigma}{\cong} S O_{3}(\mathbb{R})$. See $[\mathrm{C}$, Th. 3.1 of Ch. 10; L, Th. 3.1 of Ch. 3; Sa0; Sa1]. Two matrices above and their inverses are represented by

$$
\mu_{q}^{\varepsilon}=\mathfrak{m} \circ \sigma\left( \pm \frac{1+\varepsilon b I}{\sqrt{1+b^{2}}}\right) \quad \text { and } \quad \nu_{q}^{\delta}=\mathfrak{m} \circ \sigma\left( \pm \frac{1+\delta b K}{\sqrt{1+b^{2}}}\right),
$$

where $\varepsilon$ and $\delta$ are either -1 or 1 . For each reduced word $w$ of $\left\{\mu_{q}^{-1}, \mu_{q}, \nu_{q}^{-1}\right.$, $\left.\nu_{q}\right\}$, we define

$$
\pm H_{w}={\sqrt{1+b^{2}}}^{\sharp w} \sigma^{-1} \circ \mathfrak{m}^{-1}(w) \in Z(\mathbb{H}) /\{ \pm 1\},
$$

where $\sharp w$ is the number of occurrences of $\mu_{q}^{-1}, \mu_{q}, \nu_{q}^{-1}$, and $\nu_{q}$ in $w$ and $Z(\mathbb{H})$ is the set of quaternions whose components are all integers. The relation $H \asymp H^{\prime}$ means that $H$ and $H^{\prime}$ in $Z(\mathbb{H})$ are proportional $\bmod p$, i.e., there exists an integer $t \in\{1, \ldots, p-1\}$ such that each component of $H-t H^{\prime}$ is divisible by $p$. We consider whether $H \asymp H^{\prime}$ or not. We can choose $H_{w}$ from $\sqrt{1+b^{2}} \sigma^{-1} \circ \mathfrak{m}^{-1}(w)$ whichever you like because $H \asymp-H$.

Main result. The following two lemmas imply the main theorem of this paper which gives a free subgroup of rank 2 of $\mathrm{SO}_{3}(\mathbb{Q})$ whose non-identical elements have no fixed point on the rational sphere with radius $\sqrt{q}$.

Lemma 2. Let $w$ be a non-empty reduced word of $\left\{\mu_{q}^{-1}, \mu_{q}, \nu_{q}^{-1}, \nu_{q}\right\}$. If $w=\mu_{q}^{\varepsilon k}$ then

$$
H_{w} \asymp 1+\varepsilon b I ;
$$

if $w=\nu_{q}^{\delta l}$ then

$$
H_{w} \asymp 1+\delta b K \text {; }
$$

if $w$ has the form $\mu_{q}^{\varepsilon} \ldots \nu_{q}^{\delta}$ (i.e., w starts with $\mu_{q}^{\varepsilon}$ and ends with $\left.\nu_{q}^{\delta}\right)$ then

$$
H_{w} \asymp 1+\varepsilon b I+\varepsilon \delta J+\delta b K,
$$

where $\varepsilon$ and $\delta$ are either -1 or 1 , and $k$ and $l$ are positive integers. 
Proof. The following four equations and $1+b^{2} \equiv 0(\bmod p)$ imply this lemma:

$$
\begin{gathered}
(1+\varepsilon b I)(1+\varepsilon b I)=2(1+\varepsilon b I)-\left(1+b^{2}\right) ; \\
(1+\delta b K)(1+\delta b K)=2(1+\delta b K)-\left(1+b^{2}\right) ; \\
(1+\varepsilon b I)(1+\delta b K)=(1+\varepsilon b I+\varepsilon \delta J+\delta b K)-\left(1+b^{2}\right) \varepsilon \delta J ; \\
\left(1+\varepsilon^{\prime} b I+\varepsilon^{\prime} \delta^{\prime} J+\delta^{\prime} b K\right)(1+\varepsilon b I+\varepsilon \delta J+\delta b K) \\
=\left(1+\varepsilon^{\prime} \varepsilon+\delta^{\prime} \delta-\varepsilon^{\prime} \delta^{\prime} \varepsilon \delta\right)\left(1+\varepsilon^{\prime} b I+\varepsilon^{\prime} \delta J+\delta b K\right) \\
-\left(1+b^{2}\right)\left(\varepsilon^{\prime} \varepsilon+\delta^{\prime} \delta+\left(\varepsilon^{\prime} \delta-\delta^{\prime} \varepsilon\right) J\right) ;
\end{gathered}
$$

where $\varepsilon, \delta, \varepsilon^{\prime}$, and $\delta^{\prime}$ are either -1 or 1 .

Lemma 3. Let $w$ be a word which appeared in Lemma 2, i.e., $w=\mu_{q}^{\varepsilon k}$, $w=\nu_{q}^{\delta l}$, or $w=\mu_{q}^{\varepsilon} \ldots \nu_{q}^{\delta}$. Then $\sqrt{q}\left|\operatorname{Im} H_{w}\right|$ is irrational, where $\operatorname{Im} H_{w}$ is the imaginary part of $H_{w}$, i.e., $\operatorname{Im}(C+X I+Y J+Z K)=X I+Y J+Z K$.

Proof. It is enough to show that $q\left|\operatorname{Im} H_{w}\right|^{2}$ is a quadratic non-residue to $p$, which is a consequence of Lemma 2 , the equality $\left(\frac{q}{p}\right)=-1$, and the following three formulae:

$$
\begin{gathered}
q\left((\varepsilon t b)^{2}+0^{2}+0^{2}\right)=q t^{2} b^{2}, \\
q\left(0^{2}+0^{2}+(\delta t b)^{2}\right)=q t^{2} b^{2}, \\
q\left((\varepsilon t b)^{2}+(\varepsilon \delta t)^{2}+(\delta t b)^{2}\right)=q t^{2}\left(1+2 b^{2}\right) \equiv q t^{2} b^{2}(\bmod p),
\end{gathered}
$$

where $t=1, \ldots, p-1$.

We attain our objective from the previous lemma:

THEOREM. The rotations $\mu_{q}$ and $\nu_{q}$ generate a free group whose nontrivial elements have no fixed point on $\left(\sqrt{q} \mathbb{S}^{2}\right) \cap \mathbb{Q}^{3}$.

Proof. We only have to prove that $\operatorname{Im} H_{w}$ is non-zero and that $\sqrt{q}$. $\iota\left(\operatorname{Im} H_{w}\right) /\left|\operatorname{Im} H_{w}\right|$ does not belong to $\left(\sqrt{q} \mathbb{S}^{2}\right) \cap \mathbb{Q}^{3}$ for each reduced word, $w$, of the form $\mu_{q}^{\varepsilon} \ldots \nu_{q}^{\delta}$ (i.e., $w$ starts with $\mu_{q}^{-1}$ or $\mu_{q}$ and ends with $\nu_{q}^{-1}$ or $\nu_{q}$ ) or simply a power of $\mu_{q}$ or of $\nu_{q}$, because

$$
w \text { has a fixed point } \Leftrightarrow w^{\prime} w w^{\prime-1} \text { has a fixed point, }
$$

for an arbitrary word $w^{\prime}$ of $\left\{\mu_{q}^{-1}, \mu_{q}, \nu_{q}^{-1}, \nu_{q}\right\}$. For such a non-empty reduced word $w, \operatorname{Im} H_{w}$ is obviously non-zero and Lemma 3 implies

$$
\sqrt{q} \frac{\iota\left(\operatorname{Im} H_{w}\right)}{\left|\operatorname{Im} H_{w}\right|} \notin \mathbb{Q}^{3} \text {. }
$$

\section{References}

[C] J. W. S. Cassels, Rational Quadratic Forms, Academic Press, New York, 1978. 
$[\mathrm{H}]$ E. Hecke, Vorlesungen über die Theorie der algebraischen Zahlen, Akademische Verlagsgesellschaft, Leipzig, 1923.

[L] T. Y. Lam, Algebraic Theory of Quadratic Forms, W. A. Benjamin Inc., Massachusetts, 1973.

[M] L. J. Mordell, Diophantine Equations, Academic Press, New York, 1969.

[Sa0] K. Satô, A Hausdorff decomposition on a countable subset of $\mathbb{S}^{2}$ without the Axiom of Choice, Math. Japon. 44 (1996), 307-312.

[Sa1] K. Satô, A free group acting without fixed points on the rational unit sphere, Fund. Math. 148 (1995), 63-69.

[Sa2] - A free group of rotations with rational entries on the 3-dimensional unit sphere, Nihonkai Math. J. 8 (1997), 91-94.

[W] S. Wagon, The Banach-Tarski Paradox, Cambridge Univ. Press, Cambridge, 1985.

Department of Mathematics

Faculty of Engineering

Yokohama National University

Hodogaya, Yokohama 240, Japan

E-mail: kenzi@math.sci.ynu.ac.jp
Current address:

Department of Mathematics Faculty of Engineering

Tamagawa University

6-1-1, Tamagawa-Gakuen, Machida Tokyo 194-8610, Japan

E-mail: kenzi@eng.tamagawa.ac.jp

Received on 21.1.1997

and in revised form on 29.1.1998 